\section{Digital Instruction and the Pedagogy of Hesitation}

\section{Micah Rutenberg}

University of Tennessee, Knoxville

\section{Scott Wall}

University of Tennessee, Knoxville
"The computer has no capacity for empathy, for compassion. The computer cannot imagine the use of space. But the most important thing is that the computer cannot hesitate... Working between the mind and the hand we often hesitate, and we reveal our own answers in our hesitations." ${ }^{1}$

Juhani Pallasmaa

The reconfiguration of the world of embodied existence into a digital one over the past two decades has been a transition full of potential and possibility, but also one of pedagogical concern and uncertainty. Faculty in every school of architecture are still grappling with the challenges of building curricula that introduce digital modes of architectural production at the onset of design education while simultaneously maintaining a balanced emphasis on developing the student's spatial and experiential imagination, along with its direct translation into architectural space.

The generation of students entering architecture and design schools today are the first to be fully native to digital culture with computation, virtual existence, and access to information streams as equally relevant interfaces with the world as are the direct physical stimuli of lived experience. However, their fluency with computation does not at first appear to facilitate an innate ability to use digital tools to develop the spatial imagination or to create new synaptic connections between the spatial imagination and physical form. In fact, we often see the opposite. Rather than adding spatial depth, digital tools-everything from modes of production like laser cutters and 3D printers, to visualization tools such as Rhino, V-Ray, or Grasshopperseem to flatten space.

Obviously, the role of digital design in first-year studios continues to be debated even after decades of wide use in design. We believe that this is because - as a design culture - we have not yet come to fully reconcile the capacities of hand-craft with those afforded by the digital realm of the computer.

Our conversation about the friction between these two modes of action began with Juhani Pallasmaa's observation made in an interview in 2011. As he points out, in addition to the possibility of the so-called "happy accident", the embodied act of translation from mind to hand allows for moments of hesitation and pause. When thought and hand are misaligned, as they often are, we hesitate, and that moment of hesitation can be revelatory.

The problem, Pallasmaa argues, is that the computer cannot hesitate- it cannot feel, contemplate, or reveal. Because digital tools rely on absolutes, design happens within a highly controlled, highly governed environment that makes moments of chance and hesitation few and far between.

The deliberate, yet indeterminate process of physical making appears to be in stark contrast to the immediate, determinate, and often automatic mouse-clicks and commands by the user following a programmed logic in digital environments. So, understandably, the introduction of digital tools is often equated with the loss of a design process that values human error or the revelatory mistake. Digital tools are also faulted with making it easy for students merely execute a preestablished process, rather than seeking to challenge their 

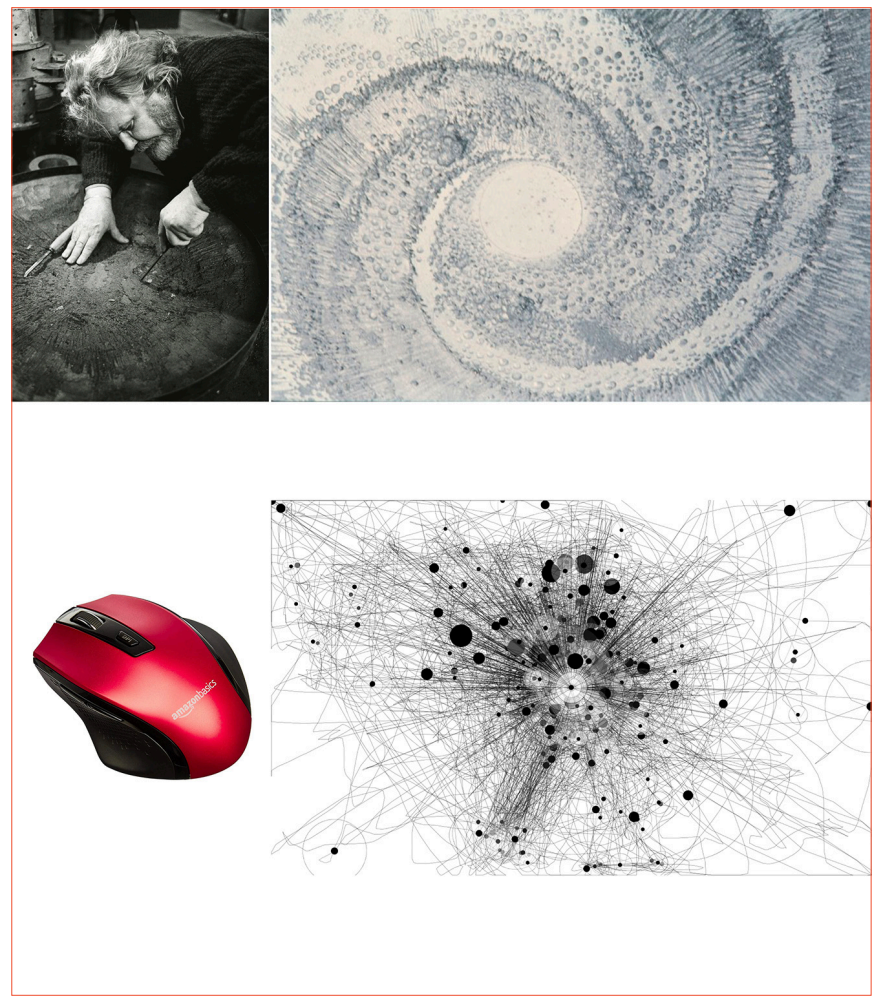

Figure 1. Top: Hand craft; Finnish designer Tapio Wirkkala, at work on the glass piece "Hole in the Ice" , 1970.

Bottom: Digital craft; Amazonbasics mouse (I) and mouse-tracking screenshot.

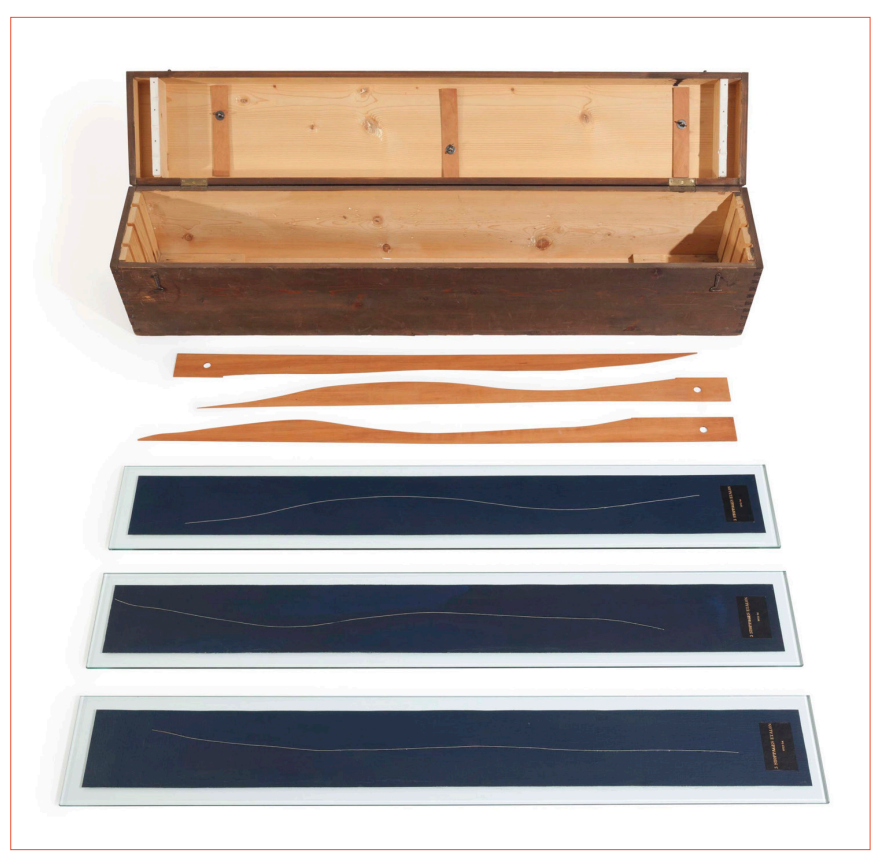

Figure 2. Marcel Duchamp, 3 Standard Stoppages, 1913-1914. conclusions or assumptions. (Figure 1)

The difficulty of digital design is that it demands explicit, predetermined decisions that eliminate opportunities for the revelatory mistake - sheer chance - that is characteristic of physical acts of making. These mistakes, as teachers know, are not errors, but moments of reflection and opportunities for invention. There is little question that digital tools have unique value, so the question we have been exploring in our first-year studios, is, how can we encourage the development and understanding of a co-extensive physical and digital design environment that values each unique mode of thinking for the opportunities each brings to the design process?

Marcel Duchamp's work from the early 20th century is instructive in this regard. As a whole the work provides a profound example and conceptual framework for "making strange" that transformed the definition of art by finding ways of challenging the overt socio-cultural controls and norms of the context in which he worked.

In particular, Duchamp's 3 Standard Stoppages (1913-1914) ${ }^{2}$ directly contests the fundamental idea of the "constant". He drops a one-meter string three times from a height of one meter, to achieve a new definition of the meter. The stoppages are inscribed in wood and collected in a box as evidence of his "redefinition" of the meter. (Figure 2)

Duchamp is working within a predetermined set of givens that become parameters for this work of art. It is a kind of pre-digital parametric design experiment. And yet, our reading of 3 Standard Stoppages is largely governed by the uncontrolled aspects of the work, meaning the way the string falls to create a new meter along with the fact that the process is open ended: he might have continued to drop an infinite number of strings to create an infinite number of stoppages. Duchamp not only destabilizes the meter per se, but makes the notion of chance the primary agent within an otherwise controlled and determinate environment.

\section{SESSION ONE: FIRST SIX WEEKS (WALL)}

Using Duchamp's destabilizing project as a pedagogical prompt, Professor Wall asked students to dismantle a "readymade" -a Valencia orange- by literally and figuratively "dissecting" it. This engaged the students in a sequence of textual, physical, and digital representations of an orange through a process of transformation. After initially

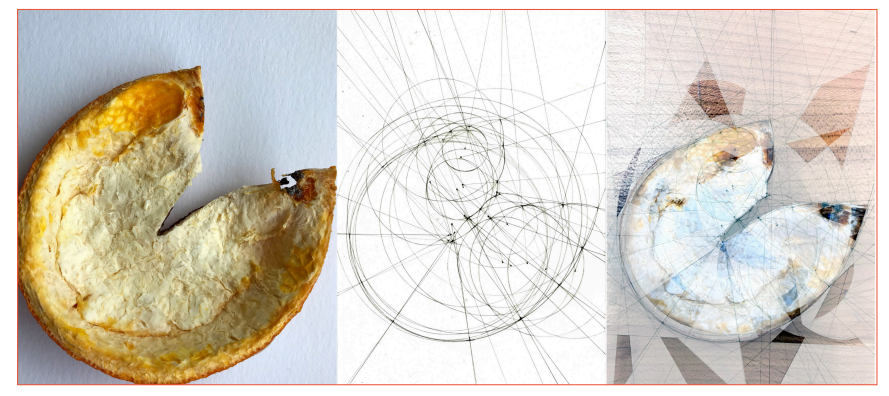

Figure 3. Original orange peel, Iterations 2 and 3 from ARC 171, Wall Studio. 


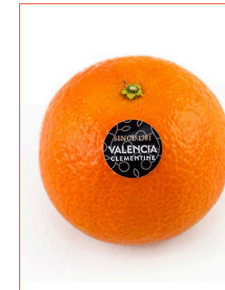

orange

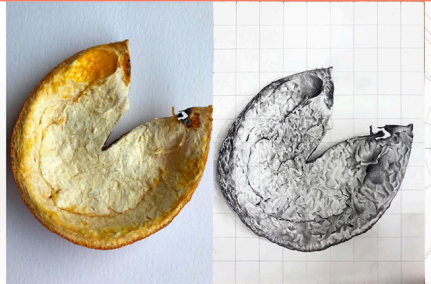

peel
11

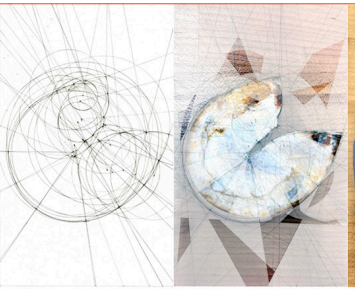

12

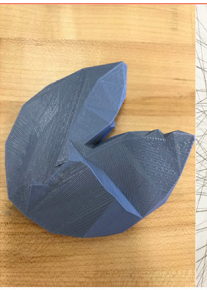

14

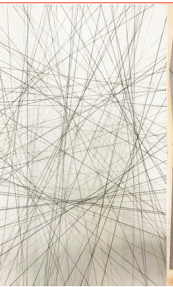

15

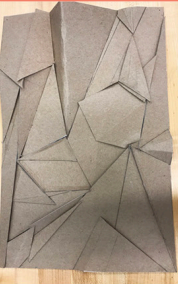

16

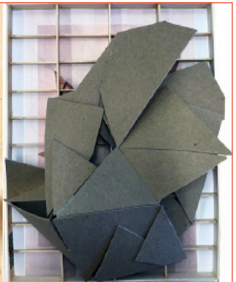

17

Cataloguing the orange: A Systematic Dictionary of Fruits, Geometry, Form, and Time. 2017

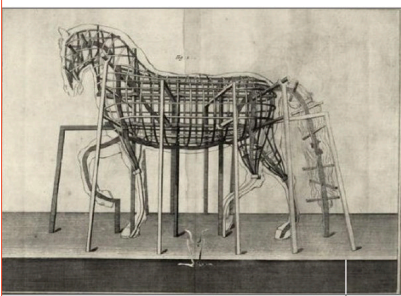

how to make an equestrian statue

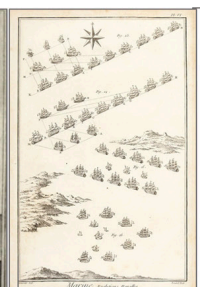

iserw

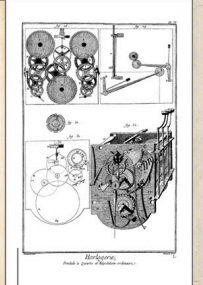

how to dismantle a watch

how to fight a naval engagement

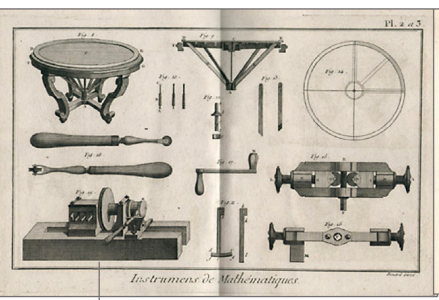

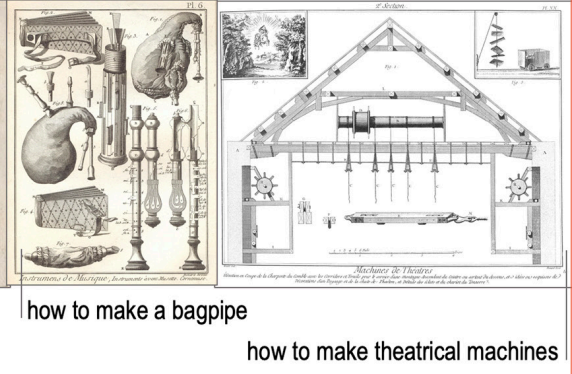

Figure 4. Top: Original orange and peel, Iterations 1-7: drawing "reality" to constructing an "aperture" from ARC 171, Wall Studio.

Bottom: Collection of plates from the Encyclopaedia, or, A Systematic Dictionary of the Sciences, Arts, and Crafts, Diderot and d'Alembert.

drawing the orange as a whole, students sectioned it, peeled it, then left the peel to dry. With each physical and digital iteration, students were asked to reconstruct the peel. The project unfolded in the following sequence of iterations.

\section{ITERATION 1: THE PEEL AND "REALITY" (PHYSICAL APPROXIMATION)}

Students employed conventional pictorial representation to describe the given: the desiccated peel of the orange. A measured grid was employed as a graphic substrate for visual and tactile observation, representing one kind of reality: made, measured, and felt by hand and eye.

\section{ITERATION 2:THE PEEL AND ABSTRACTION (GEOMETRIC APPROXIMATION)}

Students re-rationalized and abstracted the orange as a set of tangents, which revealed internal geometric relationships. They came to understand the difference between literal representation and abstract description, as well as the notion of descriptive drawing as a subjective act.

\section{ITERATION 3: THE PEEL AND DEPTH (LAYERS AND TRANSPARENCY)}

Students generated multiple digital variants based on overlay and transparency. They used the geometry of these variants to define new edges and foci within the evolving drawing. Additionally, the

drawing becomes a means of finding equivalencies between digital and physical space, employing the capacity of digital space to visibly alter the physical conditions of the drawing. (figure 3)

The ability to create versions that superimpose, reorder, clip, mask, manipulate opacity and transparency, etc., both reinforce and destabilize the idea of the orange as a constant. It has become something else. Here, chance and hesitation are not found through linear process that builds toward a final outcome, but through a rapid collection of iterations that reveal implicit qualities or patterns in-the-making.

\section{ITERATION 4. THE PEEL AND +/- (DIGITAL POSITIVE)}

Each student created a 3D scan of the peel, which generated an understanding of the relationship between the positive and negative space of the orange peel. It enabled them to see the complexity of their original abstractions (arcs, lines, layers, depth) materialized as an impenetrable object through computational processes.

\section{ITERATION 5. THE DIGITAL POSITIVE AS BOUNDARY}

Returning to the hand and visual decision-making, students drew a minimum of fifty tangents derived from the 3D print. The exercise revealed boundary logics of form and geometry, providing a set of open-ended givens for making something new - a landscape for the following iteration.

\section{ITERATION 6. THE BOUNDARY AS CONTOUR/ LANDSCAPE}

As the studio moved farther away from the peel, they were extending their awareness of the logics of design far beyond 


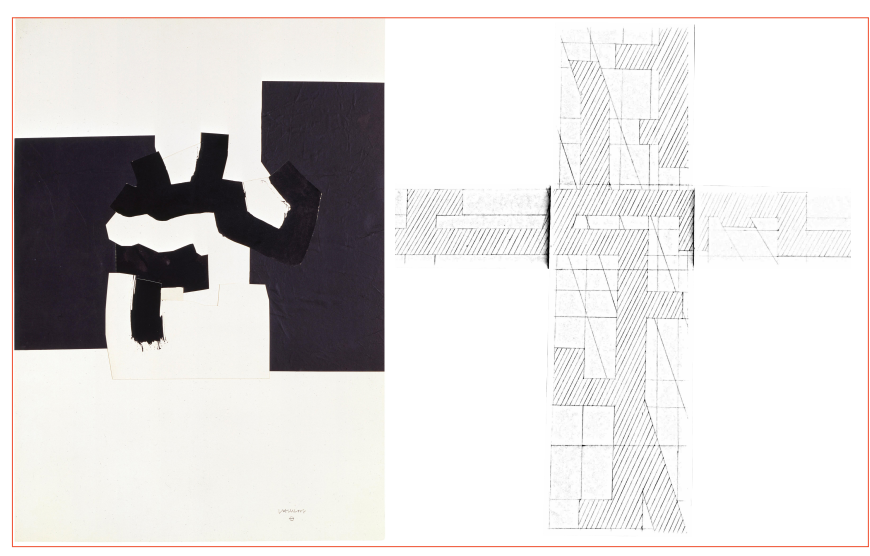

Figure 5. Left: Eduardo Chillida. Untitiled, 1970. Right: Iteration 1: Positive/ Negative Derivation Composition from ARC 171, Rutenberg Studio.
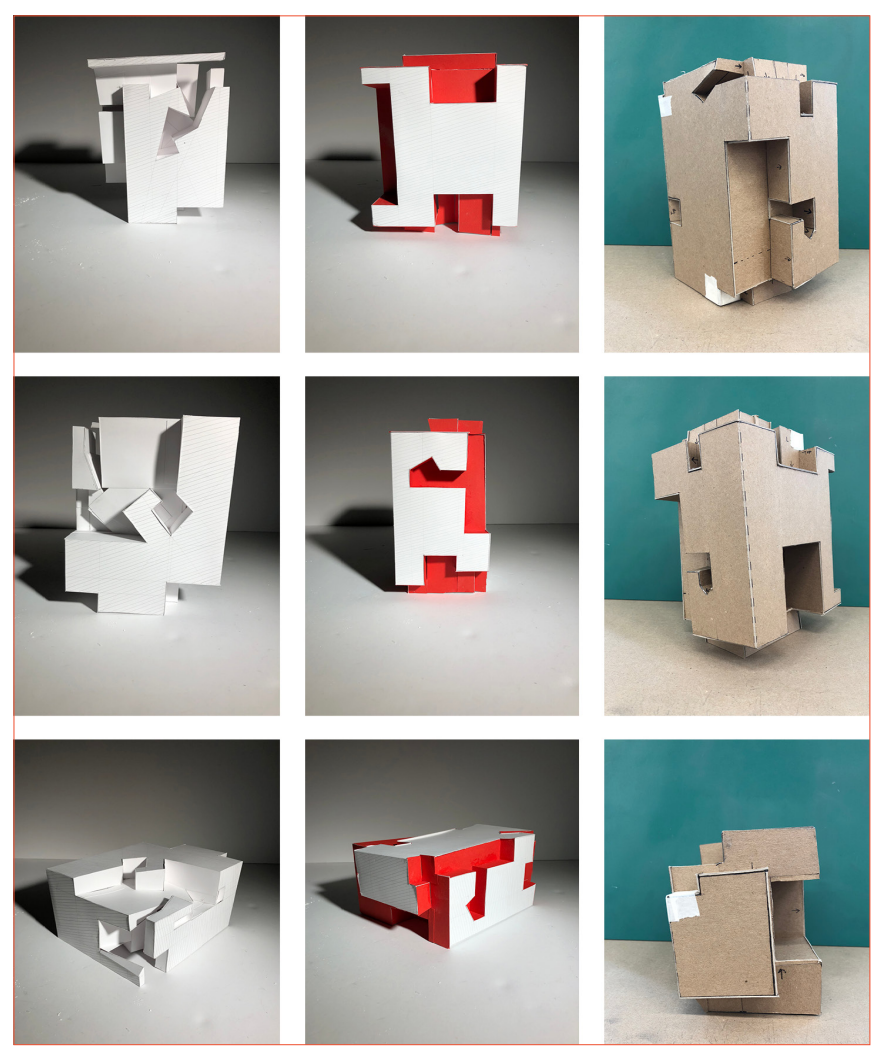

Figure 6. Iteration 3: Variable Constructs from ARC 171, Rutenberg Studio.

the origin point of an orange and its peel.

This work started with an object in the hand - an orange and its component parts. But while it was initially shaped by deliberate acts of physical making, it was not limited by it. Instead, like Diderot's Encyclopedia, it became a speculation on acts of making that precisely depict a reality, while finding revelations within the subjectivity of varied methods of cultural production and knowledge.

As a result of the process of interpreting and reinterpreting the orange through a series of representational tactics, students began to approach the iterative process as a system in which "right" and "wrong" were understood as dangerous constants. Instead, students operated within a reciprocal relationship between themselves and tools for developing formal, spatial, and material actions. This process encouraged each student to be aware of the potential of chance, and of both conscious and unconscious acts of "hesitation", as part of a design process that would move through the rest of the work. (Figure 4)

\section{SESSION TWO: SECOND SIX WEEKS (RUTENBERG)}

At this point in the semester, students were synthesizing the capacities of physical and digital methods into a process of designing embodied space and inhabitation. In the second six weeks, the "givens" provided by Professor Rutenberg were two "solid/void" paintings by Eduardo Chillida.

\section{ITERATION 1. DERIVATION (2D SOLID/VOID DIAGRAMS)}

Students reinterpreted these paintings as a series of four generative diagrams that were recombined to create a larger composition. (Figure 5)

\section{ITERATION 2. DERIVATION (3D CUTS, FOLDS, COLORS)}

Learning from the illusory qualities of Chillida's solid/void paintings, students reinterpreted their compositions by physically cutting and folding the drawing to create a 3D paper construct. They were then to strategically highlight a single plane of their model with a selected color as a way of exposing the ambiguity between 2D and $3 \mathrm{D}$ perceptions of space tudents reinterpreted these paintings as a series of four generative diagrams that were recombined to create a larger composition.

\section{ITERATION 3. VARIABLE CONSTRUCTS}

The students then modeled their paper constructs in digital space. Instead of using the digital model as a truth, or facsimile of their construct, they used the digital model to generate additional spatial variants of their project. They were asked to draw axonometric views using tone, and ideas about transparency to generate new interpretations of space.

While conventions were identified and conceptual and spatial ideas present in the original were maintained, those norms were also challenged by the interplay between legible space and visual effect. Sometimes this produced an unsettling and contradictory effect, but, ultimately, students arrived at drawings that were able to represent something already made as well as suggest possibilities for yet-to-beimagined spaces. (Figure 6) 


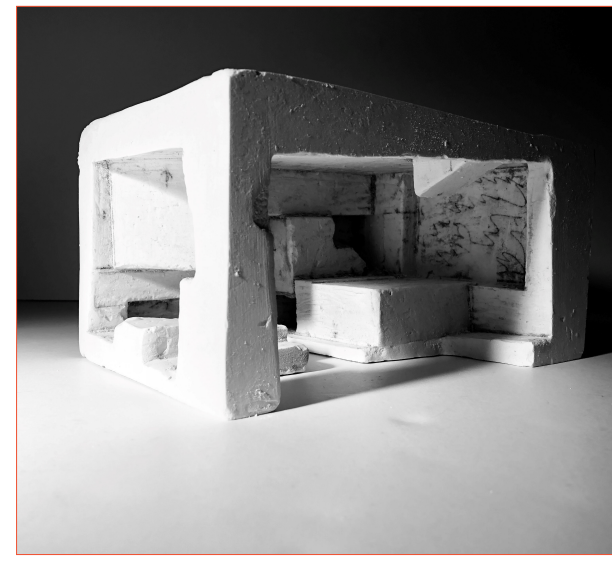

Figure 7. Iteration $3+4$ : Variable Constructs and Casting Negatives from ARC 171, Rutenberg Studio.

\section{ITERATION 4. CASTING NEGATIVES}

Subsequently, we returned to the hand and physical making. They used what they had learned to design a chipboard mold for casting spaces found within their drawings. The process of casting plaster made the negative spaces of their drawings both visible and vividly material. But, importantly, the casting process yielded unexpected material qualities and spaces that could not have been achieved or anticipated by the drawing.

Moving back and forth between the physical and digital permitted students to reflect on and recognize the importance of the decisions embedded in what they draw and make. In this process, they discovered distinct relationships between solid and void that emerged as much from the unknowns of the casting process as from their intent (Figure 7)

\section{ITERATION 5. SCALE}

Up to this point, while all of their objects were spatial, they were understood and acted upon at a 1:1 scale. To introduce the concept of perception at the scale of the body and inhabitation, students organized their casts on a grid according to a given set of rules. The configuration of their casts on a grid was defined as a new given: as a city with scale and orientation.

\section{ITERATION 6. SITE AND SITING}

Each student was provided a site within this plaster city. They were asked to imagine what the city could become and how it might be inhabited. Responses to this question played out in an iterative sequence of individual designs for a space that negotiated between the given conditions of the city, their neighbors, and their individual intent. This opened up a way to understand design as the most fundamental form of negotiation: one that requires a spatial and material understanding of both constants (grids and casts) and variables (specificity of limits of city, neighbors, and intent).
THE CITY AS ABSTRACT ORDER AND EMBODIED EXPERIENCE

Given the specificities of scale, students had to work between imagining embodied experience and the limits of the emerging spatial order. Additionally, they had to consider the woven fabric of the city from the outside, as well as learning of intended and unintended consequences of their decisions from within.

\section{THE CITY AS OPPORTUNITY}

Throughout this process, students generated digital models, which they used to study the space of the city in both perspective and section. Rather than simply illustrating what is already known, emphasis was placed on using drawing as a way to understand design decisions as actions that directly alter the shape and form of their spatial negotiation with their peers and within their city.

\section{THE CITY, BECOMING}

One of the consequences of this process was a growing awareness of the both the spatial and social complexity of any city. No city can ever be a completely "known" artifact. Instead, the city becomes understood as what all cities are: a temporary series of rules and regulations, spaces and limits, and existential conditions and concerns that are always in the process of becoming. Similarly, their models and representations are not a means of demonstrating a reality that is already known, but rather a means of interpreting, evaluating, and re-interpreting that reality. They are the means of coding, decoding and ultimately describing a new reality.

\section{THE CITY, MERGING AND EMERGING}

Our city - one that emerged as much from chance and hesitation as it did from things that are known and determinate, created an interaction between the slow modes of thought associated with the hand, and heuristic methods of reflection afforded by digital environments. (Figure 8)

\section{CONCLUSION}

The cultivation of a hybrid design process that facilitates moments of chance and hesitation allows new modes of slow thought and 
reciprocating reflection that involve, but are not subservient to computational logics. Moments of hesitation are made more urgent by the ubiquity of computation and the evolving intellectual capacities of those entering architecture schools today, whom have not yet encountered the forms of abstract spatial thought discussed in this essay. Our goal, and the task of each of us in beginning design, is to ask how we might introduce conditions of slow thinking into digital platforms, thereby balancing digital thinking with physical understanding. The iterative act is central to this endeavor.

As with Duchamp's 3 Standard Stoppages, the iterative act is not presented as a set of actions in service of reproducing a stable reality.
Instead, the promise of iteration becomes its capacity to reveal the strange truths and alternative worlds that give us pause. We frame this moment in terms of a relationship between digital and physical modes of making. One must recognize that this evolution necessitates new responses and increasingly hybrid modes of thought.
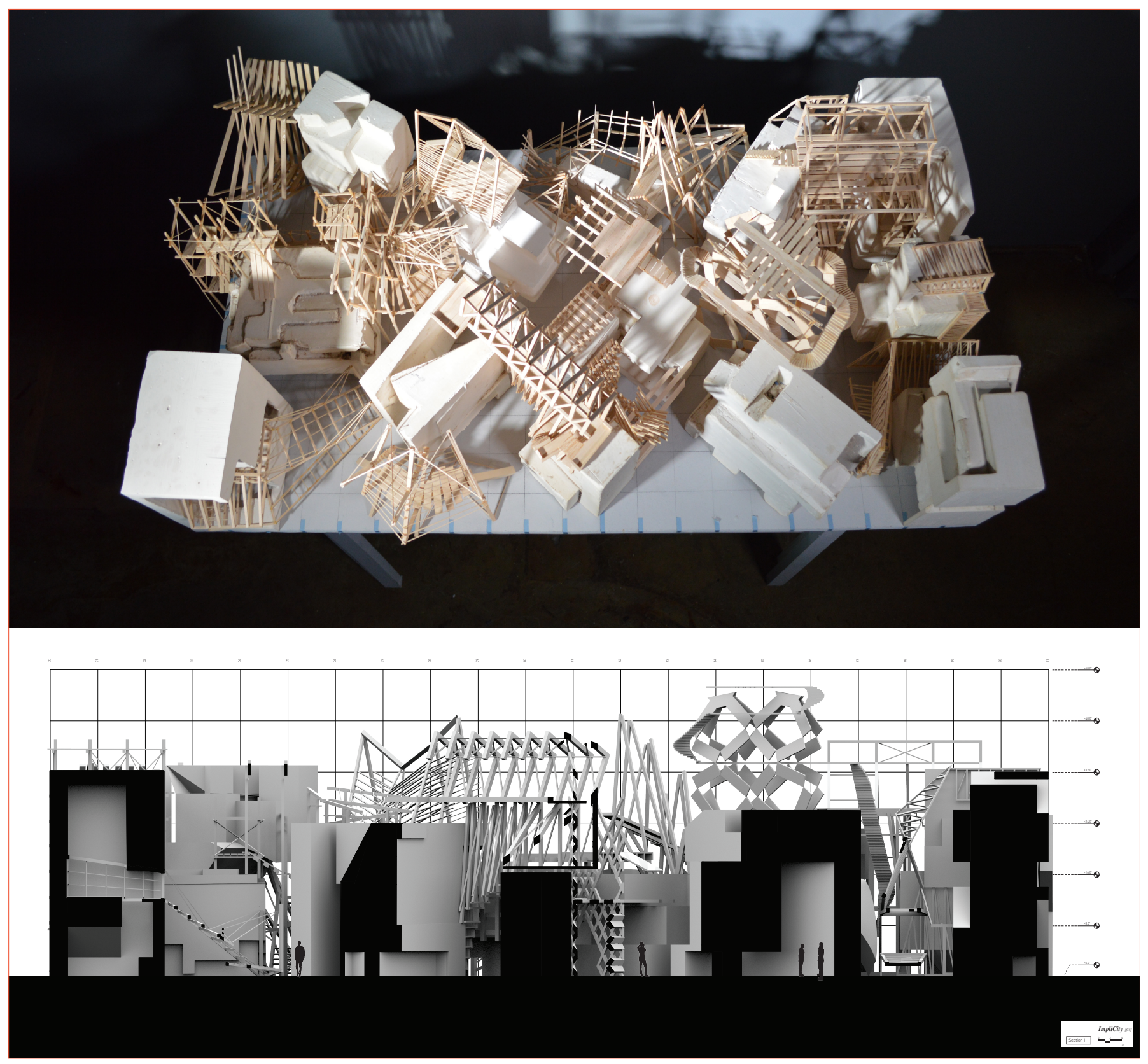

Figure 8. The City, Mergeing and Emerging from ARC 171, Rutenberg Studio. 


\section{Notes}

1. Quotation from the digitally published version of $\mathrm{A}+\mathrm{U}$,

"Rachel Hurst Talks to Juhani Pallasmaa", 27.09.2011, https://

architectureau.com/articles/juhani-pallasmaa-rachel-hurst/.

2. In the 1910's, Duchamp actively began exploring the potential of randomness, or chance, as a mechanism for both artistic expression and raising fundamental questions regarding cultural conventions and larger systems of authority.

\section{Image credits}

3. Figure 1. Top: Image courtesy of the Finnish Glass Museum, "Hole in the Ice", courtesy of the Tapio Wirkkala-Rut Bruk Foundation). Bottom: computer mouse (I) @ https://www. amazon.com/AmazonBasics-Compact-Ergonomic-WirelessScrolling, and mouse tracking screenshot (r), (https://i. imgur.com/YJeiu.png).architectureau.com/articles/ juhani-pallasmaa-rachel-hurst/.

4. Figure 2. Duchamp, Original image from Christie's Sale 14187, Post-War and Contemprorary Art Evening Sale, New York, 17 May 2017. https://www.christies.com/lotfinder Lot/marcelduchamp-1887-1968-3-standard-stoppages-6076411-d (Wall Studio. Student: Caitlin Turner, Fall 2017)

5. Figure 3. Student: Caitlin Turner. Fall, 2018.

6. Figure 4. Top: Student: Caitlin Turner, Fall 2018. Bottom: Encyclopaedia, or, A Systematic Dictionary of the Sciences, Arts, and Crafts, Diderot and d'Alembert, editors, published in the fi e volumes of Diderot Encyclopedia: Complete Illustrations 1762-1777, New York: Harry Abrams, 1978.

7. Figure 5. Left: Eduardo Chillida. Untitled, 1970. http:// catalogue.colognefine rt.com. Right: Student: Ashley Cardosi, Fall 2018.

8. Figure 6. Student: John Schumacher, Fall 2018.

9. Figure 7. Student: John Schumacheri, Fall 2018.

10. Figure 8. Collective model and drawings. Fall 2018. 